\title{
Fixed Points and Stability of a Class of Integrodifferential Equations
}

\author{
Dingheng Pi \\ School of Mathematical Sciences, Huaqiao University, Quanzhou, Fujian 362021, China \\ Correspondence should be addressed to Dingheng Pi; pidh@hqu.edu.cn \\ Received 11 April 2014; Accepted 30 May 2014; Published 18 June 2014 \\ Academic Editor: Yuxin Zhao
}

Copyright (C) 2014 Dingheng Pi. This is an open access article distributed under the Creative Commons Attribution License, which permits unrestricted use, distribution, and reproduction in any medium, provided the original work is properly cited.

We study a class of integrodifferential functional differential equations $\ddot{x}+f(t, x, \dot{x}) \dot{x}+\sum_{j=1}^{N} \int_{t-r_{j}(t)}^{t} a_{j}(t, s) g_{j}(s, x(s)) d s=0$ with variable delay. By using the fixed point theory, we establish necessary and sufficient conditions ensuring that the zero solution of this equation is asymptotically stable.

\section{Introduction}

It is well known that differential equations have many applications in engineering, control theory, neural networks, biology, and so on. The stability of the solution of functional differential equations has drawn a lot of attention for many years. Liapunov's direct method has been widely applied to study the stability problems for a long time; see, for example, [1-3]. Nowadays, many authors have applied the fixed points theory to study the stability of solution of functional differential equations; some authors even use fixed point theory to study stability conditions for stochastic equations with delay; see, for example, [4-7] and the references therein.

Burton studied stability of a nonconvolution equation

$$
\dot{x}=-\int_{t-r}^{t} a(t, s) g(x(s)) d s
$$

where $r$ was a positive constant. He obtained sufficient conditions ensuring that the zero solution was asymptotically stable via fixed point theorem; see [8].

Becker and Burton studied the following differential equation:

$$
\dot{x}=-\int_{t-r(t)}^{t} a(t, s) g(x(s)) d s
$$

with variable delay and its special case

$$
\dot{x}=-\int_{t-r}^{t} a(t-s) g(x(s)) d s,
$$

for $r$, is a positive constant, $t \geq 0$, where function $r(t)$ : $[0, \infty) \rightarrow[0, \infty)$. They found sufficient conditions ensuring that the zero solution was asymptotically stable by changing the supremum metric to an exponentially weighted metric. Moreover, they hoped to reduce condition that the function $t-r(t)$ was strictly increasing; see [9]. Jin and Luo reduced this condition in their paper. Moreover, they established necessary and sufficient conditions that could ensure that the zero solution of this equation is asymptotically stable; see, for example, [10].

Ardjouni et al. studied the stability of the following neutral integrodifferential equation:

$$
\dot{x}(t)=-\sum_{j=1}^{N} \int_{t-\tau_{j}(t)}^{t} a_{j}(t, s) x(s) d s+\sum_{j=1}^{N} c_{j}(t) x^{\prime}\left(t-\tau_{j}(t)\right)
$$

via fixed point theorem. Also $m_{j}(0)=\inf \left\{t-\tau_{j}(t): t \geq 0\right\}$. Their main result was as follows.

Theorem A. Suppose that $\tau_{j}$ is twice differentiable and $\tau_{j}^{\prime}(t) \neq 1$ for all $t \in R^{+}$and there exist continuous functions 
$h_{j}:\left[m_{j}(0), \infty\right) \rightarrow R$ for $j=1,2, \ldots, N$ and a constant $\alpha \in(0,1)$ such that, for $t \geq 0$,

(i) $\lim \inf _{t \rightarrow \infty} \int_{0}^{t} H(s) d s>-\infty$,

(ii)

$$
\begin{aligned}
& \sum_{j=1}^{N}\left|\frac{c_{j}(t)}{1-\tau_{j}^{\prime}(t)}\right|+\sum_{j=1}^{N} \int_{t-\tau_{j}(t)}^{t}\left|h_{j}(s)+B_{j}(t, s)\right| d s \\
& +\sum_{j=1}^{N} \int_{0}^{t} e^{-\int_{s}^{t} H(u) d u}\left|h_{j}\left(s-\tau_{j}(s)\right)\left(1-\tau_{j}^{\prime}(s)-r_{j}(s)\right)\right| d s \\
& +\sum_{j=1}^{N} \int_{0}^{t} e^{-\int_{s}^{t} H(u) d u}|H(s)| \\
& \quad \times \int_{s-\tau_{j}(s)}^{s}\left|h_{j}(u)+B_{j}(s, u)\right| d u d s \leq \alpha,
\end{aligned}
$$

where

$$
\begin{gathered}
H(t)=\sum_{j=1}^{N} h_{j}(t), \\
r_{j}(t)=\frac{\left[c_{j}(t) H(t)+c_{j}^{\prime}(t)\right]\left(1-\tau_{j}^{\prime}(t)\right)+c_{j}(t) \tau_{j}^{\prime \prime}(t)}{\left(1-\tau_{j}^{\prime}(t)\right)^{2}}, \\
B_{j}(t, s)=\int_{t}^{s} a_{j}(u, s) d u, \\
B_{j}\left(t, t-\tau_{j}(t)\right)=\int_{t}^{t-\tau_{j}(t)} a_{j}\left(u, t-\tau_{j}(t)\right) d u .
\end{gathered}
$$

Then the zero solution is asymptotically stable if and only if

$$
\int_{0}^{t} H(s) d s \longrightarrow \infty \quad \text { as } t \longrightarrow \infty \text {. }
$$

They improved the results of [9]; see [11]. Other results on fixed points and stability properties in equations with variable delays can be found in [5].

Recently, Zhao and Yuan investigate the stability of a generalized Volterra-Levin equation:

$$
\begin{gathered}
\dot{x}=-\int_{t-L}^{t} p(s-t) g(s, x(s)) d s, \quad t>t_{0}, \\
x(t)=\psi(t), \quad t_{0}-L \leq t \leq t_{0} .
\end{gathered}
$$

They give new conditions on the stability of zero solution of this equation; their conditions are different from [5]; see, for example, [12].

The stability of second order equation has always been hot issue of research. Levin and Nohel [13] studied the global asymptotic stability of a class of nonlinear systems:

$$
\ddot{x}(t)+h(t, x, \dot{x}) \dot{x}+f(x)=e(t) .
$$

They obtained asymptotic stability by constructing proper Lyapunov function.

Burton [14] considered the following equation with delay:

$$
\ddot{x}+f(t, x, \dot{x}) \dot{x}+b(t) g(x(t-L))=0,
$$

where $L$ is a positive constant, and obtained some sufficient conditions under which each solution $x(t)$ satisfies $(x(t), \dot{x}(t)) \rightarrow 0$ via the fixed point theorem.

We generalized the above equation to an equation with a variable delay [15] as follows:

$$
\ddot{x}+f(t, x, \dot{x}) \dot{x}+b(t) g(x(t-\tau(t)))=0,
$$

and obtained some results on asymptotic stability of the zero solution. Before we introduce our new results we recall the main results in [15]. There are basic assumptions on the delay function $\tau(t):(\mathscr{A}) t-\tau(t)$ which is strictly increasing and $\lim _{t \rightarrow \infty} t-\tau(t)=\infty$. $p(t)$ is the inverse of $t-\tau(t)$. Moreover $0 \leq b(t) \leq M$ for some constant $M>0$.

The main results in [15] can be stated as follows.

Theorem B. Suppose $(\mathscr{A})$ and the following conditions hold.

(i) There exists a constant $l>0$ such that $g(x)$ satisfies the Lipschitz condition on $[-l, l]$. The function $g(x)$ is odd and is strictly increasing on $[-l, l]$, and $x-g(x)$ is nondecreasing on $[0, l]$.

(ii) There exist an $\alpha \in(0,1)$ and a continuous function $a(t):[0, \infty) \rightarrow[0, \infty)$ such that $f(t, x, y) \geq a(t)$ for $t \geq 0, x \in \mathbb{R}, y \in \mathbb{R}, \int_{0}^{\infty} a(t) d t=\infty$, and

$$
\begin{aligned}
& 2 \sup _{t \geq 0} \int_{t}^{p(t)} \int_{0}^{\infty} e^{-\int_{s}^{w+s} a(v) d v} b(s) d w d s \\
& \quad+2 \sup _{t \geq 0} \int_{0}^{t} \int_{t-s}^{\infty} e^{-\int_{s}^{w+s} a(v) d v} b(s) d w d s \leq \alpha .
\end{aligned}
$$

(iii) There exist constants $a_{0}>0$ and $Q>0$ such that, for each $t \geq 0$, if $J \geq Q$, then

$$
\int_{t}^{t+J} a(v) d v \geq a_{0} J .
$$

(iv) There exist continuous functions $F: \mathbb{R} \times \mathbb{R} \rightarrow[0, \infty)$ and $c(t):[0, \infty) \rightarrow[0, \infty)$ such that, for all $t \geq 0$, $x \in \mathbb{R}, y \in \mathbb{R}, f(t, x, y) \leq F(x, y) c(t)$. The function $g^{\prime}(x)$ is continuous on $[-l, l], g^{\prime}(0) \neq 0$.

If, for each $\gamma>0, \iint_{0}^{\infty} e^{-\int_{s}^{w+s} \gamma c(v)} b(s) d w d s=\infty$, then the zero solution of (11) is asymptotically stable.

In [16], we have studied the following equation:

$$
\ddot{x}+f(t, x, \dot{x}) \dot{x}+\int_{t-r(t)}^{t} a(t, s) g(x(s)) d s=0
$$

with variable delay. By using the fixed point theory, we obtain conditions which ensure that the zero solution of this equation is stable under an exponentially weighted metric. 
Moreover, we establish necessary and sufficient conditions ensuring that the zero solution is asymptotically stable.

In this paper we consider

$$
\ddot{x}+f(t, x, \dot{x}) \dot{x}+\sum_{j=1}^{N} \int_{t-r_{j}(t)}^{t} a_{j}(t, s) g_{j}(s, x(s)) d s=0
$$

for $t \geq 0$, where functions $r_{j}(t):[0, \infty) \rightarrow[0, \infty), a_{j}(t, s):$ $[0, \infty) \times[-r(0), \infty) \rightarrow R, g_{j}:[-r(0), \infty) \times R \rightarrow R$, and $f: R \times R \times R \rightarrow R^{+}$are all continuous, where $R^{+}=[0,+\infty)$. We assume that $t-r_{j}(t) \rightarrow \infty$ as $t \rightarrow \infty . j=1,2, \ldots, N$.

For each $t_{0} \geq 0$, define $m_{j}\left(t_{0}\right)=\inf \left\{s-r_{j}(s): s \geq t_{0}\right\}$, $j=1,2, \ldots, N$. Let $m\left(t_{0}\right)=\min \left\{m_{1}\left(t_{0}\right), \ldots, m_{N}\left(t_{0}\right)\right\}$. Also $C\left(t_{0}\right)=C\left(\left[m\left(t_{0}\right), t_{0}\right], R\right)$ is a function space endowed with function norm $\|\cdot\|$, where $\|\psi\|=\sup \left\{|\psi(s)|: m\left(t_{0}\right) \leq s \leq t_{0}\right\}$. We will also use $\|\phi\|$ to express the supremum on $\left[m\left(t_{0}\right), \infty\right)$ later. It is well known that, in [2], for a given continuous function $\phi$, there exists a solution of (15) on an interval $\left[t_{0}, T\right)$; if the solution remains bounded, then $T=\infty$. We denote by $(x(t), y(t))$ the solution $\left(x\left(t, t_{0}, \phi\right), y\left(t, t_{0}, \phi\right)\right)$. Denote by $A(t):=f(t, x(t), y(t))$.

We can write (15) in an equivalent form as

$$
\begin{gathered}
\dot{x}=y, \\
\dot{y}=-A(t) y-\sum_{j=1}^{N} \int_{t-r_{j}(t)}^{t} a_{j}(t, s) g_{j}(s, x(s)) d s .
\end{gathered}
$$

We will give a necessary and sufficient condition ensuring that the zero solution of this equation is asymptotically stable. To our knowledge, there are few results about its stability. This paper is organized as follows. In the next section we will state our main results. Their proofs will be given in Sections 3 and 4 .

\section{Statement of Main Results}

First of all, we consider a special case of (15). We consider the following equation:

$$
\ddot{x}+f(t, x, \dot{x}) \dot{x}+\int_{t-L}^{t} p(s-t) g(s, x(s)) d s=0 .
$$

Rewrite this equation as

$$
\begin{gathered}
\dot{x}=y, \\
\dot{y}=-A(t) y-\int_{t-L}^{t} p(s-t) g(s, x(s)) d s .
\end{gathered}
$$

Our main results are as follows.

Theorem 1. Assume that $h(t) \in C\left(R^{+}, R^{+}\right)$and the following conditions hold.

(i) Consider that $\int_{-L}^{0} p(s) d s=1$. There exist a constant $\lambda>0$ and a function $Q(x) \in C\left(R, R^{+}\right)$such that, for $\forall x, y_{1}, y_{2} \in R,\left|g\left(x, y_{1}\right)-g\left(x, y_{2}\right)\right| \leq Q(x)\left|y_{1}-y_{2}\right|$ and the inequality $\sup _{t \geq 0} \int_{-L}^{0}|p(s)| \int_{t-L}^{t+s} Q(u) d u d s \leq \lambda$ hold. There exists a constant $K>0$ such that $Q(t) \leq K$. (ii) There exist $\alpha \in(0,1)$ and a continuous function $A_{1}(t)$ : $[0, \infty) \rightarrow[0, \infty)$ such that $f(t, x, y) \geq A_{1}(t)$ for $t \geq$ 0 . For $t \geq 0, x \in R, y \in R$

$$
\begin{gathered}
\int_{0}^{t} e^{-\int_{u}^{t} h(s) d s} \int_{0}^{u} e^{-\int_{s}^{u} A_{1}(v) d v} Q(s-L) d s d u \\
\quad+2 \lambda \int_{0}^{t} e^{-\int_{u}^{t} h(s) d s} d u \leq \alpha
\end{gathered}
$$

(iii) There exist constants $a_{0}>0$ and $Q_{0}>0$ such that, for each $t \geq 0$, if $J \geq Q_{0}$, then

$$
\int_{t}^{t+J} A_{1}(s) d s \geq a_{0} J
$$

Then the zero solution of (17) is stable.

In addition, we have the following.

Theorem 2. Assume that $\lim _{t \rightarrow \infty} t-r_{j}(t)=\infty, r_{j}(t)$ is differentiable, $j=1,2, \ldots, N$, and there exist some functions $h_{j}(t) \in C\left(R^{+}, R^{+}\right)$such that for $t \geq 0$ the following conditions hold.

(i) There exist a constant $\Gamma>0$ and some functions $Q_{j}(x) \in C\left(R, R^{+}\right)$such that, for $\forall x, y_{1}, y_{2} \in R$,

$$
\begin{gathered}
\left|g_{j}\left(x, y_{1}\right)-g_{j}\left(x, y_{2}\right)\right| \leq Q_{j}(x)\left|y_{1}-y_{2}\right|, \quad j=1,2, \ldots, N, \\
\sup _{t \geq 0} \sum_{j=1}^{N} \int_{t-r_{j}(t)}^{t}\left(\int_{t}^{u}\left|a_{j}(v, t)\right| d v\right) Q_{j}(u) d u \leq \Gamma .
\end{gathered}
$$

There exists a constant $K^{*}>0$ such that $Q_{j}(t) \leq K^{*}$, $j=1,2, \ldots, N$.

(ii) Let $H(t)=\sum_{j=1}^{N} h_{j}(t)$. There exist a constant $\alpha \in(0,1)$ and a continuous function $A_{1}(t): R^{+} \rightarrow R^{+}$such that $f(t, x, y) \geq A_{1}(t)$. For $\forall t \geq 0, x \in R, y \in R$,

$$
\begin{aligned}
& 2 \sum_{j=1}^{N} \int_{t-r_{j}(t)}^{t} h_{j}(v) d v+2 \Gamma \int_{0}^{t} e^{-\int_{u}^{t} H(v) d v} d u \\
& \quad+\sum_{j=1}^{N} \int_{0}^{t} e^{-\int_{s}^{t} H(u) d u} h_{j}\left(s-r_{j}(s)\right)\left|1-r_{j}^{\prime}(s)\right| d s \\
& \quad+\sum_{j=1}^{N} \int_{0}^{t} e^{-\int_{u}^{t} H(v) d v}\left(\int_{0}^{u} e^{-\int_{s}^{u} A_{1}(v) d v} K^{*}\left|W_{j}(s)\right| d s\right) d u \leq \alpha,
\end{aligned}
$$

where $W_{j}(s)=\left(1-r_{j}^{\prime}(s)\right) \int_{s}^{s-r_{j}(s)} a_{j}\left(v, s-r_{j}(s)\right) d v$.

(iii) There exist constants $a_{0}>0$ and $Q_{0}>0$ such that, for each $t \geq 0$, if $J \geq Q_{0}$, then

$$
\int_{t}^{t+J} A_{1}(s) d s \geq a_{0} J
$$

Then the zero solution of (15) is asymptotically stable if and only if

$$
\int_{0}^{t} H(s) d s \longrightarrow \infty \quad \text { as } t \longrightarrow \infty .
$$




\section{Proof of Theorem 1}

In this section, we will prove Theorem 1 by applying the fixed point theory. We will give the expression of the solution of the related equation. The following result can be found in [12].

Lemma 3. Let the function $p:[-L, 0] \rightarrow R, g \in C(R \times R, R)$, $\int_{-L}^{0} p(s) d s=1$. Then

$$
\dot{x}(t)=-\int_{t-L}^{t} p(s-t) g(s, x(s)) d s
$$

is equivalent to

$$
\begin{aligned}
\dot{x}(t)= & -g(t-L, x(t-L)) \\
& +\frac{d}{d t} \int_{-L}^{0} p(s) \int_{t+s}^{t-L} g(u, x(u)) d u d s .
\end{aligned}
$$

Lemma 4. Let $\psi:[-L, 0] \rightarrow R$ be a given continuous function; if $(x(t), y(t))$ is the solution of (17) satisfying $x(t)=$ $\psi(t), t \in[-L, 0]$, and $y(0)=\dot{x}(0)$, then $x(t)$ is the solution of the following integral equation:

$x(t)$

$$
\begin{gathered}
=\psi(0) e^{-\int_{0}^{t} h(s) d s}+\int_{0}^{t} e^{-\int_{u}^{t} h(s) d s} B(u) d u \\
-\int_{0}^{t} e^{-\int_{u}^{t} h(s) d s}\left(\int_{0}^{u} e^{-\int_{s}^{u} A(v) d v} g(s-L, x(s-L)) d s\right) d u \\
+\int_{0}^{t} e^{-\int_{u}^{t} h(s) d s}\left(e^{-\int_{0}^{u} A(v) d v}\right. \\
\left.\quad \times \int_{-L}^{0} p(v) \int_{v}^{-L} g(w, \psi(w)) d w d v\right) d u \\
-\int_{0}^{t} e^{-\int_{u}^{t} h(s) d s}\left(\int_{-L}^{0} p(v) \int_{u+v}^{u-L} g(w, x(w)) d w d v\right) d u \\
-\int_{0}^{t} e^{-\int_{u}^{t} h(s) d s}\left[\int_{0}^{u} e^{-\int_{s}^{u} A(v) d v} A(s)\right. \\
\times\left(\int_{-L}^{0} p(v)\right. \\
\left.\left.\times \int_{s+v}^{s-L} g(w, x(w)) d w d v\right) d s\right] d u,
\end{gathered}
$$

where $B(t)=\dot{x}(0) e^{-\int_{0}^{t} A(s) d s}$.

Proof. We apply the variation of parameters formula to the second equation of (18); we obtain

$$
\begin{aligned}
\dot{x}(t)= & \dot{x}(0) e^{-\int_{0}^{t} A(s) d s} \\
& -\int_{0}^{t} e^{-\int_{s}^{t} A(v) d v}\left(\int_{s-L}^{s} p(v-s) g(v, x(v)) d v\right) d s .
\end{aligned}
$$

By Lemma 3, (28) can be written as

$$
\begin{aligned}
\dot{x}(t)= & B(t)-\int_{0}^{t} e^{-\int_{s}^{t} A(v) d v} g(s-L, x(s-L)) d s \\
& +\int_{0}^{t} e^{-\int_{s}^{t} A(v) d v} \\
& \quad \times\left(\frac{d}{d s} \int_{-L}^{0} p(s) \int_{s+v}^{s-L} g(u, x(u)) d u d v\right) d s ;
\end{aligned}
$$

multiplying both sides of the above equation by $e^{\int_{0}^{t} h(s) d s}$, we see that

$$
\begin{aligned}
& x(t) \\
& =\psi(0) e^{-\int_{0}^{t} h(s) d s}+\int_{0}^{t} e^{-\int_{u}^{t} h(s) d s} B(u) d u \\
& -\int_{0}^{t} e^{-\int_{u}^{t} h(s) d s}\left(\int_{0}^{u} e^{-\int_{s}^{u} A(v) d v} g(s-L, x(s-L)) d s\right) d u \\
& +\int_{0}^{t} e^{-\int_{u}^{t} h(s) d s} \\
& \times\left[\int_{0}^{u} e^{-\int_{s}^{u} A(v) d v}\right. \\
& \times\left(\frac{d}{d s} \int_{-L}^{0} p(s)\right. \\
& \left.\left.\times \int_{s+v}^{s-L} g(w, x(w)) d w d v\right) d s\right] d u .
\end{aligned}
$$

If we integrate the last term by parts, we have

$x(t)$

$$
\begin{gathered}
=\psi(0) e^{-\int_{0}^{t} h(s) d s}+\int_{0}^{t} e^{-\int_{u}^{t} h(s) d s} B(u) d u \\
-\int_{0}^{t} e^{-\int_{u}^{t} h(s) d s}\left(\int_{0}^{u} e^{-\int_{s}^{u} A(v) d v} g(s-L, x(s-L)) d s\right) d u \\
+\int_{0}^{t} e^{-\int_{u}^{t} h(s) d s}\left(e^{-\int_{0}^{u} A(v) d v}\right. \\
\left.\quad \times \int_{-L}^{0} p(v) \int_{v}^{-L} g(w, \psi(w)) d w d v\right) d u \\
-\int_{0}^{t} e^{-\int_{u}^{t} h(s) d s}\left(\int_{-L}^{0} p(v) \int_{u+v}^{u-L} g(w, x(w)) d w d v\right) d u \\
-\int_{0}^{t} e^{-\int_{u}^{t} h(s) d s} \\
\times\left[\int_{0}^{u} e^{-\int_{s}^{u} A(v) d v} A(s)\right. \\
\times\left(\int_{-L}^{0} p(v)\right. \\
\left.\left.\times \int_{s+v}^{s-L} g(w, x(w)) d w d v\right) d s\right] d u .
\end{gathered}
$$


Let $(C,\|\cdot\|)$ be the Banach space of bounded continuous functions on $[-L, \infty)$ with the supremum norm. For a given continuous initial function $\psi:[-L, 0] \rightarrow R$, define the set $C_{\psi} \subset C$ by

$$
C_{\psi}=\{\phi:[-L, \infty) \longrightarrow R \mid \phi \in C, \phi(t)=\psi(t), t \in[-L, 0]\}
$$

and its subset

$$
\begin{gathered}
C_{\psi}^{l}=\{\phi:[-L, \infty) \longrightarrow R \mid \phi \in C, \phi(t)=\psi(t), \\
t \in[-L, 0],|\phi(t)| \leq l, t>0\},
\end{gathered}
$$

where $\psi:[-L, 0] \rightarrow[-l, l]$ is a given initial function and $l$ is a positive constant. Let $P_{1}$ be a mapping defined on $C_{\psi}^{l}$ as follows: for $\phi \in C_{\psi}^{l}$, if $t \in[-L, 0],\left(P_{1} \phi\right)(t)=\psi(t)$. If $t>0$,

$$
\begin{gathered}
\left(P_{1} \phi\right)(t) \\
=\psi(0) e^{-\int_{0}^{t} h(s) d s}+\int_{0}^{t} e^{-\int_{u}^{t} h(s) d s} B(u) d u \\
-\int_{0}^{t} e^{-\int_{u}^{t} h(s) d s} \int_{0}^{u} e^{-\int_{s}^{u} A(v) d v} g(s-L, \phi(s-L)) d s d u \\
+\int_{0}^{t} e^{-\int_{u}^{t} h(s) d s}\left(e^{-\int_{0}^{u} A(v) d v}\right. \\
\left.\quad \times \int_{-L}^{0} p(v) \int_{v}^{-L} g(w, \psi(w)) d w d v\right) d u \\
-\int_{0}^{t} e^{-\int_{u}^{t} h(s) d s}\left(\int_{-L}^{0} p(v) \int_{u+v}^{u-L} g(w, \phi(w)) d w d v\right) d u \\
-\int_{0}^{t} e^{-\int_{u}^{t} h(s) d s}\left[\int_{0}^{u} e^{-\int_{s}^{u} A(v) d v} A(s)\right. \\
\times\left(\int_{-L}^{0} p(v)\right. \\
\left.\left.\times \int_{s+v}^{s-L} g(w, \phi(w)) d w d v\right) d s\right] d u .
\end{gathered}
$$

Easy calculation shows that

$$
\begin{aligned}
& \left|\left(P_{1} \phi\right)(t)-\left(P_{1} \eta\right)(t)\right| \\
& \leq \int_{0}^{t} e^{-\int_{u}^{t} h(s) d s} \int_{0}^{u} e^{-\int_{s}^{u} A(v) d v} \mid g(s-L, \phi(s-L)) \\
& \quad-g(s-L, \eta(s-L)) \mid d s d u \\
& \quad \int_{0}^{t} e^{-\int_{u}^{t} h(s) d s}\left(\int_{-L}^{0} p(v) \int_{u-L}^{u+v} \mid g(w, \phi(w))\right. \\
& -g(w, \eta(w)) \mid d w d v) d u
\end{aligned}
$$

$$
\begin{aligned}
& +\int_{0}^{t} e^{-\int_{u}^{t} h(s) d s} \\
& \times\left(\int_{0}^{u} e^{-\int_{s}^{u} A(v) d v} A(s)\right. \\
& \quad \times\left(\int_{-L}^{0} p(v)\right. \\
& \quad \times \int_{s-L}^{s+v} \mid g(w, \phi(w)) \\
& \quad-g(w, \eta(w)) \mid d w d v) d s) d u .
\end{aligned}
$$

We can easily obtain that $\left(P_{1} \phi\right)(t)$ is a contraction mapping by using condition (ii). We continue to prove Theorem 1 . Choose $\psi:[-L, 0] \rightarrow R$ and $\dot{x}(0)$ such that

$$
\left(Q_{0}+\frac{e^{-a_{0} Q_{0}}}{a_{0}}\right)|\dot{x}(0)|+\delta+\lambda \delta\left(Q_{0}+\frac{e^{-a_{0} Q_{0}}}{a_{0}}\right) \leq(1-\alpha) l .
$$

Condition (i) implies that $g(t, 0)=0$, since $g(t, x)$ satisfies the Lipschitz condition with respect to $x$, such that $\delta$ exists and $\delta<l$. By the expression of $\left(P_{1} \phi\right)(t)$, condition (ii), and condition (iii) of Theorem 1, we have

$$
\begin{aligned}
\int_{0}^{t} e^{-\int_{0}^{u} A(s) d s} d u & =\int_{0}^{\mathrm{Q}_{0}} e^{-\int_{0}^{u} A(s) d s} d u+\int_{\mathrm{Q}_{0}}^{t} e^{-\int_{0}^{u} A(s) d s} d u \\
& \leq \int_{0}^{\mathrm{Q}_{0}} d u+\int_{\mathrm{Q}_{0}}^{t} e^{-a_{0} u} d u \\
& \leq Q_{0}+\frac{e^{-a_{0} \mathrm{Q}_{0}}}{a_{0}} .
\end{aligned}
$$

According to condition (ii) and (36), we have

$$
\left|\left(P_{1} \phi\right)(t)\right| \leq(1-\alpha) l+\alpha l=l .
$$

Note that if $t \in[-L, 0]$, then $\left(P_{1} \phi\right)(t)=\psi(t)$. We obtain that $\left|\left(P_{1} \phi\right)(t)\right| \leq l, t \in[-L, \infty)$. Thus $P_{1}: C_{\psi}^{l} \rightarrow C_{\psi}^{l}$. Since we have proved that $P_{1}$ is a contraction mapping, hence $P_{1}$ has a unique fixed point $x(t)$ and $|x(t)| \leq l$.

Recalling (28), we have

$$
\begin{aligned}
|y(t)| \leq & |\dot{x}(0)| e^{-\int_{0}^{t} A(s) d s} \\
& +\int_{0}^{t} e^{-\int_{s}^{t} A(v) d v} \int_{s-L}^{s}|p(s-v)||g(v, x(v))| d v d s ;
\end{aligned}
$$

then

$$
\begin{aligned}
|y(t)| & \leq|\dot{x}(0)|+K l \cdot \int_{0}^{t} e^{-\int_{u}^{t} A(s) d s} d u \\
& <l\left(1+K\left(Q_{0}+\frac{e^{-a_{0} Q_{0}}}{a_{0}}\right)\right) .
\end{aligned}
$$


It follows that

$$
|x(t)|+|y(t)|<l\left(2+K\left(Q_{0}+\frac{e^{-a_{0} Q_{0}}}{a_{0}}\right)\right) .
$$

For any given $\epsilon>0$; we only need to replace $\epsilon$ by $l$. Then we obtain the stability of zero solution. This completes the proof of Theorem 1 .

\section{Proof of Theorem 2}

In this section, we will prove Theorem 2 . First of all, we will obtain a useful expression for the solution of (15) in the following proof.

We apply the variation of parameters formula to the second equation of (15); then

$$
\begin{aligned}
\dot{x}(t)=B_{1}(t)-\sum_{j=1}^{N} \int_{t_{0}}^{t} e^{-\int_{s}^{t} A(v) d v} & \\
& \times\left(\int_{s-r_{j}(s)}^{s} a_{j}(s, v) g_{j}(v, x(v)) d v\right) d s,
\end{aligned}
$$

where $B_{1}(t)=\dot{x}\left(t_{0}\right) e^{-\int_{t_{0}}^{t} A(s) d s}$.

Lemma 5. The equation

$$
\dot{x}(t)=-\sum_{j=1}^{N} \int_{t-r_{j}(t)}^{t} a_{j}(t, s) g_{j}(s, x(s)) d s
$$

is equivalent to

$$
\begin{aligned}
& \dot{x}(t) \\
& =\sum_{j=1}^{N} C_{j}\left(t, t-r_{j}(t)\right)\left(1-r_{j}^{\prime}(t)\right) g_{j}\left(t-r_{j}(t), x\left(t-r_{j}(t)\right)\right) \\
& \quad+\sum_{j=1}^{N} \frac{d}{d t} \int_{t-r_{j}(t)}^{t} C_{j}(t, s) g_{j}(s, x(s)) d s,
\end{aligned}
$$

where

$$
\begin{gathered}
C_{j}(t, s)=\int_{t}^{s} a_{j}(u, s) d u \\
C_{j}\left(t, t-r_{j}(t)\right)=\int_{t}^{t-r_{j}(t)} a_{j}\left(u, t-r_{j}(t)\right) d u .
\end{gathered}
$$

Proof. Differentiating the integral term in (44), we have

$$
\begin{aligned}
& \frac{d}{d t} \int_{t-r_{j}(t)}^{t} C_{j}(t, s) g_{j}(s, x(s)) d s \\
& =\int_{t-r_{j}(t)}^{t} \frac{\partial}{\partial t} C_{j}(t, s) g_{j}(s, x(s)) d s+C_{j}(t, t) g_{j}(t, x(t)) \\
& \quad-C_{j}\left(t, t-r_{j}(t)\right) g_{j}\left(t-r_{j}(t), x\left(t-r_{j}(t)\right)\right) \\
& \quad \times\left(1-r_{j}^{\prime}(t)\right) .
\end{aligned}
$$

It follows that if $C_{j}(t, t)=0,(\partial / \partial t) C_{j}(t, s)=-a_{j}(t, s)$, then (43) is equivalent to (44). Easy calculation shows that

$$
\begin{gathered}
C_{j}(t, s)=\int_{t}^{s} a_{j}(u, s) d u \\
C_{j}\left(t, t-r_{j}(t)\right)=\int_{t}^{t-r_{j}(t)} a_{j}\left(u, t-r_{j}(t)\right) d u .
\end{gathered}
$$

By Lemma 5, (42) can be written as

$$
\begin{gathered}
\dot{x}(t)=B_{1}(t)+\sum_{j=1}^{N} \int_{t_{0}}^{t} e^{-\int_{s}^{t} A(v) d v} \\
\times\left(\frac{d}{d s} \int_{s-r_{j}(s)}^{s} C_{j}(s, v)\right. \\
\left.\times g_{j}(v, x(v)) d v\right) d s \\
+\sum_{j=1}^{N} \int_{t_{0}}^{t} e^{-\int_{s}^{t} A(v) d v}\left(C_{j}\left(s, s-r_{j}(s)\right)\left(1-r_{j}^{\prime}(s)\right)\right. \\
\left.\quad \times g_{j}\left(s-r_{j}(s), x\left(s-r_{j}(s)\right)\right)\right) d s .
\end{gathered}
$$

We define

$$
\begin{gathered}
C_{\psi}^{0}=\left\{\phi:\left[m\left(t_{0}\right), \infty\right) \longrightarrow R \mid \phi \in C, \phi(t)=\psi(t),\right. \\
\left.t \in\left[m\left(t_{0}\right), t_{0}\right], \phi(t) \longrightarrow 0 \text { as } t \longrightarrow \infty\right\} .
\end{gathered}
$$

Multiplying both sides of the above equation by $e^{\int_{t_{0}}^{t} H(u) d u}$, easy calculation shows that

$x(t)$

$$
\begin{aligned}
& =\psi\left(t_{0}\right) e^{-\int_{t_{0}}^{t} H(u) d u}+\int_{t_{0}}^{t} e^{-\int_{s}^{t} H(u) d u} B_{1}(s) d s \\
& +\int_{t_{0}}^{t} e^{-\int_{s}^{t} H(u) d u} H(s) x(s) d s \\
& +\sum_{j=1}^{N} \int_{t_{0}}^{t} e^{-\int_{u}^{t} H(v) d v} \\
& \times\left(\int_{t_{0}}^{u} e^{-\int_{s}^{u} A(v) d v} \frac{d}{d s}\right. \\
& \left.\times \int_{s-r_{j}(s)}^{s} C_{j}(s, v) g_{j}(v, x(v)) d v\right) d s d u \\
& +\sum_{j=1}^{N} \int_{t_{0}}^{t} e^{-\int_{u}^{t} H(v) d v}\left(\int_{t_{0}}^{u} e^{-\int_{s}^{u} A(v) d v} W_{j}(s)\right. \\
& \left.\times g_{j}\left(s-r_{j}(s), x\left(s-r_{j}(s)\right)\right) d s\right) d u,
\end{aligned}
$$


where $W_{j}(s)=C_{j}\left(s, s-r_{j}(s)\right)\left(1-r_{j}^{\prime}(s)\right)$. Performing an integration by parts, then

$x(t)$

$=\psi\left(t_{0}\right) e^{-\int_{t_{0}}^{t} H(s) d s}+\int_{t_{0}}^{t} e^{-\int_{s}^{t} H(u) d u} B_{1}(s) d s$

$+\sum_{j=1}^{N} \int_{t-r_{j}(t)}^{t} h_{j}(v) x(v) d v$

$-\sum_{j=1}^{N} \int_{t_{0}-r_{j}\left(t_{0}\right)}^{t_{0}} h_{j}(u) \psi(u) d u \cdot e^{-\int_{t_{0}}^{t} H(u) d u}$

$-\sum_{j=1}^{N} \int_{t_{0}}^{t}\left(\int_{s-r_{j}(s)}^{s} h_{j}(v) x(v) d v\right) e^{-\int_{s}^{t} H(u) d u} H(s) d s$

$+\sum_{j=1}^{N} \int_{t_{0}}^{t} e^{-\int_{s}^{t} H(u) d u} h_{j}\left(s-r_{j}(s)\right) x\left(s-r_{j}(s)\right)$

$$
\times\left(1-r_{j}^{\prime}(s)\right) d s
$$

$+\sum_{j=1}^{N} \int_{t_{0}}^{t} e^{-\int_{u}^{t} H(v) d v}\left(\int_{u-r_{j}(u)}^{u} C_{j}(u, v) g_{j}(v, x(v)) d v\right) d u$

$-\sum_{j=1}^{N} \int_{t_{0}}^{t} e^{-\int_{u}^{t} H(v) d v}\left[\int_{t_{0}}^{u} e^{-\int_{t_{0}}^{u} A(v) d v}\right.$

$$
\times\left(\int_{t_{0}-r_{j}\left(t_{0}\right)}^{t_{0}} C_{j}\left(t_{0}, v\right)\right.
$$

$$
\left.\left.\times g_{j}(v, \psi(v)) d v\right) d s\right] d u
$$

$-\sum_{j=1}^{N} \int_{t_{0}}^{t} e^{-\int_{s}^{t} H(u) d u}\left(\int_{t_{0}}^{s} e^{-\int_{v}^{s} A(r) d r} A(v)\right.$

$$
\times \int_{v-r_{j}(v)}^{v} C_{j}(v, u)
$$$$
\left.\times g_{j}(u, x(u)) d u d v\right) d s
$$

$+\sum_{j=1}^{N} \int_{t_{0}}^{t} e^{-\int_{u}^{t} H(v) d v}\left(\int_{t_{0}}^{u} e^{-\int_{s}^{u} A(v) d v} W_{j}(s)\right.$

$$
\left.\times g_{j}\left(s-r_{j}(s), x\left(s-r_{j}(s)\right)\right) d s\right) d u .
$$

Let $P_{2}$ be a mapping defined on $C_{\psi}^{0}$ as follows: for $\phi \in C_{\psi}$, if $t \in\left[m\left(t_{0}\right), t_{0}\right],\left(P_{2} \phi\right)(t)=\psi(t)$. If $t>t_{0}$, we define

$\left(P_{2} \phi\right)(t)$

$=\psi\left(t_{0}\right) e^{-\int_{t_{0}}^{t} H(s) d s}+\int_{t_{0}}^{t} e^{-\int_{s}^{t} H(u) d u} B_{1}(s) d s$
$+\sum_{j=1}^{N} \int_{t-r_{j}(t)}^{t} h_{j}(v) \phi(v) d v$

$-\sum_{j=1}^{N} \int_{t_{0}-r_{j}\left(t_{0}\right)}^{t_{0}} h_{j}(u) \psi(u) d u \cdot e^{-\int_{t_{0}}^{t} H(u) d u}$

$-\sum_{j=1}^{N} \int_{t_{0}}^{t}\left(\int_{s-r_{j}(s)}^{s} h_{j}(v) \phi(v) d v\right) e^{-\int_{s}^{t} H(u) d u} H(s) d s$

$+\sum_{j=1}^{N} \int_{t_{0}}^{t} e^{-\int_{s}^{t} H(u) d u} h_{j}(s-r(s)) \phi\left(s-r_{j}(s)\right)$

$\times\left(1-r_{j}^{\prime}(s)\right) d s$

$+\sum_{j=1}^{N} \int_{t_{0}}^{t} e^{-\int_{u}^{t} H(v) d v}\left(\int_{u-r_{j}(u)}^{u} C_{j}(u, v) g_{j}(v, \phi(v)) d v\right) d u$

$-\sum_{j=1}^{N} \int_{t_{0}}^{t} e^{-\int_{u}^{t} H(v) d v}$

$\times\left[\int_{t_{0}}^{u} e^{-\int_{t_{0}}^{u} A(v) d v}\right.$

$\times\left(\int_{t_{0}-r_{j}\left(t_{0}\right)}^{t_{0}} C_{j}\left(t_{0}, v\right)\right.$

$\left.\left.\times g_{j}(v, \psi(v)) d v\right) d s\right] d u$

$-\sum_{j=1}^{N} \int_{t_{0}}^{t} e^{-\int_{s}^{t} H(u) d u}$

$\times\left(\int_{t_{0}}^{s} e^{-\int_{v}^{s} A(r) d r} A(v)\right.$

$\left.\times \int_{v-r_{j}(v)}^{v} C_{j}(v, u) g_{j}(u, \phi(u)) d u d v\right) d s$

$+\sum_{j=1}^{N} \int_{t_{0}}^{t} e^{-\int_{u}^{t} H(v) d v}$

$\times\left(\int_{t_{0}}^{u} e^{-\int_{s}^{u} A(v) d v}\right.$

$\left.\times W_{j}(s) g_{j}\left(s-r_{j}(s), \phi\left(s-r_{j}(s)\right)\right) d s\right) d u$.

If $\phi \in C_{\psi}^{0}$, since $\int_{0}^{t} H(s) d s \rightarrow \infty$ as $t \rightarrow \infty, t_{0} \geq 0$, hence $\int_{t_{0}}^{t} H(s) d s \rightarrow \infty$ as $t \rightarrow \infty$. The first term and fourth term of $\left(P_{2} \phi\right)(t) \rightarrow 0$, as $t \rightarrow \infty$.

Note that

$\int_{t_{0}}^{t} e^{-\int_{u}^{t} H(s) d s} B_{1}(u) d u=\dot{x}\left(t_{0}\right) \int_{t_{0}}^{t} e^{-\int_{u}^{t} H(s) d s} e^{-\int_{t_{0}}^{u} A(s) d s} d u$. 
Since $A(t)=f(t, x(t), y(t)) \geq A_{1}(t) \geq 0$, then

$$
\int_{t_{0}}^{t} e^{-\int_{u}^{t} H(s) d s} e^{-\int_{t_{0}}^{u} A(s) d s} d u \leq \int_{t_{0}}^{t} e^{-\int_{t_{0}}^{u} A(s) d s} d u .
$$

For a given $\epsilon>0$, there exists $T_{1}>Q_{0}+t_{0}$ such that $e^{a_{0} t_{0}-a_{0} T}<a_{0} \epsilon$. For $T_{1}<t$ and $t_{0}<Q_{0}<T_{1} \leq u$, we have

$$
\begin{aligned}
\int_{T_{1}}^{t} e^{-\int_{t_{0}}^{u} A(s) d s} d u & \leq \int_{T_{1}}^{t} e^{-a_{0}\left(u-t_{0}\right)} d u=\frac{e^{a_{0} t_{0}}}{a_{0}}\left(e^{-a_{0} T_{1}}-e^{-a_{0} t}\right) \\
& <\frac{e^{a_{0} t_{0}-a_{0} T_{1}}}{a_{0}}<\epsilon .
\end{aligned}
$$

For $t>T_{1}$, we have

$$
\begin{aligned}
& \int_{t_{0}}^{T_{1}} e^{-\int_{u}^{t} H(s) d s} e^{-\int_{t_{0}}^{u} A(s) d s} d u \\
& \quad=e^{-\int_{T_{1}}^{t} H(s) d s} \cdot \int_{t_{0}}^{T_{1}} e^{-\int_{u}^{T_{1}} H(s) d s} e^{-\int_{t_{0}}^{u} A(s) d s} d u .
\end{aligned}
$$

The second term of $\left(P_{2} \phi\right)(t) \rightarrow 0$, as $t \rightarrow \infty$.

Note that $\int_{t_{0}-r_{j}\left(t_{0}\right)}^{t_{0}} C_{j}\left(t_{0}, v\right) g_{j}(v, \psi(v)) d v$ is bounded; we can check the eighth term of $\left(P_{2} \phi\right)(t) \rightarrow 0$, as $t \rightarrow \infty$ similarly.

Then we can easily check that $P_{2}$ is a contraction mapping on $C_{\psi}^{0}$ by using condition (ii). By the contraction mapping principle, $P_{2}$ has a unique fixed point $x(t)$ in $C_{\psi}^{0}$. Also $x(t) \rightarrow$ 0 as $t \rightarrow+\infty$.

In order to obtain the asymptotic stability, we still need to show that the zero solution is stable. Let $\epsilon>0$ be given, we choose $\psi:\left[m\left(t_{0}\right), t_{0}\right] \rightarrow R$ and $\dot{x}\left(t_{0}\right)$ such that

$$
\begin{aligned}
\delta+\left(Q_{0}\right. & \left.+\frac{e^{-a_{0} Q_{0}}}{a_{0}}\right)\left|\dot{x}\left(t_{0}\right)\right| \\
& +\sum_{j=1}^{N} \int_{t_{0}-r_{j}\left(t_{0}\right)}^{t_{0}} h_{j}(s) d s \cdot \delta+\alpha \Gamma\left(Q+\frac{e^{-a_{0} Q}}{a_{0}}\right) \delta \\
\leq & (1-\alpha) \epsilon .
\end{aligned}
$$

Then we have

$$
\begin{aligned}
|x(t)| \leq & \delta+\int_{t_{0}}^{t} e^{-\int_{u}^{t} H(s) d s}\left|\dot{x}\left(t_{0}\right)\right| e^{-\int_{t_{0}}^{u} A(s) d s} d u \\
& +\sum_{j=1}^{N} \int_{t_{0}-r_{j}\left(t_{0}\right)}^{t_{0}} h_{j}(s) d s \cdot \delta+\alpha \Gamma\left(Q+\frac{e^{-a_{0} Q}}{a_{0}}\right) \delta+\alpha \epsilon .
\end{aligned}
$$

Easy calculation shows that

$$
\begin{aligned}
\int_{t_{0}}^{t} e^{-\int_{t_{0}}^{u} A(s) d s} d u & \leq \int_{t_{0}}^{t_{0}+Q_{0}} d u+\int_{t_{0}+Q_{0}}^{t} e^{-a_{0}\left(u-t_{0}\right)} d u \\
& \leq Q_{0}+\frac{e^{-a_{0} Q_{0}}}{a_{0}} .
\end{aligned}
$$

Hence

$$
|x(t)| \leq \epsilon
$$

Therefore

$$
\begin{aligned}
& |\dot{x}(t)| \\
& \leq\left|\dot{x}\left(t_{0}\right)\right|+\sum_{j=1}^{N} \int_{t_{0}}^{t} e^{-\int_{s}^{t} A(v) d v} \\
& \quad \times\left(\int_{s-r_{j}(s)}^{s}\left|a_{j}(s, v) g_{j}(v, x(v))\right| d v\right) d s .
\end{aligned}
$$

It follows that

$$
|x(t)|+|y(t)|<\epsilon\left(2+N K^{*}\left(Q_{0}+\frac{e^{-a_{0} Q_{0}}}{a_{0}}\right)\right) .
$$

Therefore the zero solution is stable; since we have obtained that $x(t) \rightarrow 0$ as $t \rightarrow \infty$, it follows that the zero solution is asymptotically stable.

A necessary condition is as follows. For each $t_{0} \geq 0$, we denote that $M=\sup _{t \geq 0}\left\{e^{-\int_{0}^{t} H(s) d s}\right\}$. We will prove that $\int_{0}^{\infty} H(s) d s=\infty$ by way of contradiction. If $\int_{0}^{\infty} H(s) d s<\infty$, since $H(t) \geq 0, \exists$ being a sequence $\left\{t_{n}\right\}, t_{n} \rightarrow \infty$ as $n \rightarrow \infty$ such that $\lim _{n \rightarrow \infty} \int_{0}^{t_{n}} H(s) d s=\sigma$, where $\sigma$ is a finite number. Choose $\eta$ such that $-\eta \leq \int_{0}^{t_{n}} H(s) d s \leq \eta$ holds, for all $n \geq 1$.

Denote that

$$
\begin{aligned}
\omega(u) \triangleq & H(u) \sum_{j=1}^{N} \int_{u-r_{j}(u)}^{u}\left|h_{j}(v)\right| d v \\
& +\sum_{j=1}^{N}\left|h_{j}\left(s-r_{j}(s)\right)\left(1-r_{j}^{\prime}(s)\right)\right| \\
& +\sum_{j=1}^{N} \int_{t_{0}}^{u} e^{-\int_{v}^{u} A(r) d r} A(v) \\
& +\sum_{j=1}^{N} \int_{0}^{t} e^{-\int_{u}^{t} H(v) d v}\left(\int_{0}^{u} e^{-\int_{s}^{u} A_{1}(v) d v} K^{*}\right. \\
& +\sum_{j=1}^{N} \int_{u-r_{j}(u)}^{u}\left|C_{j}(u, v)\right| Q_{j}(v) d v d u,
\end{aligned}
$$

where $W_{j}(s)=\left(1-r_{j}^{\prime}(s)\right) \int_{s}^{s-r_{j}(s)} a_{j}\left(v, s-r_{j}(s)\right) d v$.

By conditions of Theorem 2, we have

$$
0 \leq \int_{0}^{t_{n}} \omega(u) e^{-\int_{u}^{t_{n}} H(s) d s} d u \leq \alpha .
$$


Then

$$
\int_{0}^{t_{n}} \omega(u) e^{\int_{0}^{u} H(s) d s} d u \leq \alpha e^{\int_{0}^{t_{n}} H(s) d s} \leq \alpha e^{\eta}
$$

Thus the sequence $\left\{\int_{0}^{t_{n}} \omega(u) e_{0}^{u} H(s) d s d u\right\}$ is bounded; there exists a convergent subsequence; we assume that $\lim _{n \rightarrow \infty} \int_{0}^{t_{n}} \omega(u) e^{\int_{0}^{u} H(s) d s} d u=I, I \geq 0$. We can choose a positive integer $\bar{k}$ large enough such that

$$
\int_{t_{\bar{k}}}^{t_{n}} \omega(u) e^{\int_{0}^{u} H(s) d s} d u<\frac{\delta_{0}}{8 M}
$$

for $\forall n \geq \bar{k}$, where $0<\delta_{0}\left(\delta_{0}<\epsilon\right)$ is satisfying

$$
\begin{aligned}
\delta_{0}+ & \max \left\{Q_{0}+\frac{e^{-a_{0} Q_{0}}}{a_{0}}, 1\right\} \delta_{0}+\delta_{0} \sum_{j=1}^{N} \int_{t_{\bar{k}}-r_{j}\left(t_{\bar{k}}\right)}^{t_{\bar{k}}} h_{j}(s) d s \\
+ & \alpha \Gamma\left(Q_{0}+\frac{e^{-a_{0} Q_{0}}}{a_{0}}\right) \delta_{0} \leq(1-\alpha) .
\end{aligned}
$$

Now we consider the solution $x(t)=x\left(t, \psi, \dot{x}\left(t_{\bar{k}}\right)\right)$ of (11) which satisfies

$$
\begin{gathered}
\psi\left(t_{\bar{k}}\right)=\frac{3 \delta_{0}}{4}, \quad \dot{x}\left(t_{\bar{k}}\right)=\frac{\delta_{0}}{4}, \\
|\psi(s)|+|\dot{x}(s)| \leq \delta_{0}, \quad s \leq t_{\bar{k}} .
\end{gathered}
$$

We can obtain that $|x(t)| \leq 1$ by a similar argument with (60) if we replace $\epsilon$ by 1 . Consider

$$
\begin{aligned}
\psi\left(t_{\bar{k}}\right) & -\sum_{j=1}^{N} \int_{t_{\bar{k}} r_{j}\left(t_{\bar{k}}\right)}^{t_{\bar{k}}} h_{j}(s) \psi(s) d s \\
& \geq \psi\left(t_{\bar{k}}\right)-\sum_{j=1}^{N} \int_{t_{\bar{k}}-r_{j}\left(t_{\bar{k}}\right)}^{t_{\bar{k}}} h_{j}(s)|\psi(s)| d s \\
& \geq \frac{3 \delta_{0}}{4}-\frac{\delta_{0}}{2}=\frac{\delta_{0}}{4} .
\end{aligned}
$$

By (27) and $|x(t)| \leq 1$, if $t_{n} \geq t_{\bar{k}}$, we have

$$
\begin{aligned}
& \left|x\left(t_{n}\right)-\sum_{j=1}^{N} \int_{t_{n}-r_{j}\left(t_{n}\right)}^{t_{n}} h_{j}(s) x(s) d s\right| \\
& \geq \mid \psi\left(t_{\bar{k}}\right) e^{-\int_{t_{\bar{k}}}^{t_{n}} H(s) d s}+\int_{t_{\bar{k}}}^{t_{n}} e^{-\int_{u}^{t_{n}} H(s) d s} B(u) d u \\
& \quad-\sum_{j=1}^{N} \int_{t_{\bar{k}-r_{j}\left(t_{\bar{k}}\right)}^{t_{\bar{k}}}}^{t_{j}} h_{j}(s) \psi(s) d s \cdot e^{-\int_{\bar{k}_{\bar{k}}}^{t_{n}} H(u) d u \mid} \\
& \quad-\left|\int_{t_{\bar{k}}}^{t_{n}} e^{-\int_{u}^{t_{n}} H(s) d s} \omega(u) d u\right| .
\end{aligned}
$$

This implies that

$$
\begin{gathered}
\left|x\left(t_{n}\right)-\sum_{j=1}^{N} \int_{t_{n}-r_{j}\left(t_{n}\right)}^{t_{n}} h_{j}(s) x(s) d s\right| \\
\geq e^{-\int_{t_{\bar{k}}}^{t_{n}} H(s) d s}\left[\left|\frac{\delta_{0}}{4}+\int_{t_{\bar{k}}}^{t_{n}} e^{\int_{t_{\bar{k}}}^{u} H(s) d s} \dot{x}\left(t_{\bar{k}}\right) e^{\int_{t_{\bar{k}}}^{u} A(s) d s} d u\right|\right. \\
\left.-\int_{t_{\bar{k}}}^{t_{n}} \omega(u) e^{\int_{t_{\bar{k}}}^{u} H(s) d s} d u\right] .
\end{gathered}
$$

Note that $\dot{x}\left(t_{\bar{k}}\right)=\delta_{0} / 4>0$; it follows that

$$
\begin{gathered}
\left|x\left(t_{n}\right)-\sum_{j=1}^{N} \int_{t_{n}-r_{j}\left(t_{n}\right)}^{t_{n}} h_{j}(s) x(s) d s\right| \\
\geq e^{-\int_{t_{\bar{k}}}^{t_{n}} h(s) d s}\left[\frac{\delta_{0}}{4}-e^{-\int_{0}^{t_{\bar{k}}} H(s) d s}\right. \\
\left.\cdot \int_{t_{\bar{k}}}^{t_{n}} \omega(u) e^{\int_{0}^{u} H(s) d s} d u\right] \\
\geq \frac{\delta_{0}}{8} e^{-\int_{t_{\bar{k}}}^{t_{n}} H(s) d s} \geq \frac{\delta_{0}}{8} e^{-2 \eta}>0 .
\end{gathered}
$$

If the zero solution $x(t)$ is asymptotically stable, then $x(t) \rightarrow 0$ as $t \rightarrow \infty$. By the mean value theorem and condition (ii), we have

$$
\begin{aligned}
\left|\int_{t_{n}-r_{j}\left(t_{n}\right)}^{t_{n}} h_{j}(s) x(s) d s\right| & =\left|(x(\varpi)) \int_{t_{n}-r_{j}\left(t_{n}\right)}^{t_{n}} h_{j}(s) d s\right| \\
& \leq|x(\varpi)| .
\end{aligned}
$$

Thus

$$
\sum_{j=1}^{N} \int_{t_{n}-r_{j}\left(t_{n}\right)}^{t_{n}} h_{j}(s) x(s) d s \longrightarrow 0 \quad \text { as } t_{n} \longrightarrow \infty .
$$

Consequently,

$$
\lim _{t_{n} \rightarrow \infty}\left(x\left(t_{n}\right)-\sum_{j=1}^{N} \int_{t_{n}-r_{j}\left(t_{n}\right)}^{t_{n}} h_{j}(s) x(s) d s\right)=0 .
$$

This is a contradiction to (72).

Hence

$$
\int_{0}^{\infty} H(s) d s=\infty .
$$

This completes the proof of Theorem 2 .

\section{Conflict of Interests}

The author declares that there is no conflict of interests regarding the publication of this paper.

\section{Acknowledgments}

This work is partially supported by NNSF of China, Grants 11226145 and 11271046, and a Research Foundation of Huaqiao University 12BS112. 


\section{References}

[1] T. A. Burton, Stability and Periodic Solutions of Ordinary and Functional-Differential Equations, Academic Press, Orlando, Fla, USA, 1985.

[2] J. K. Hale, Theory of Functional Differential Equations, Springer, New York, NY, USA, 1977.

[3] J. Levin and A. Nohel, “On a nonlinear delay equation," Journal of Mathematical Analysis and Applications, vol. 8, pp. 31-44, 1964.

[4] A. Ardjouni and A. Djoudi, "Fixed points and stability in linear neutral differential equations with variable delays," Nonlinear Analysis: Theory, Methods \& Applications, vol. 74, no. 6, pp. 2062-2070, 2011.

[5] T. A. Burton, Stability by Fixed Point Theory for Functional Differential Equations, Dover, Mineola, NY, USA, 2006.

[6] C. H. Jin and J. W. Luo, "Fixed points and stability in neutral differential equations with variable delays," Proceedings of the American Mathematical Society, vol. 136, no. 3, pp. 909-918, 2008.

[7] D. Zhao, S. Yuan, and T. Zhang, "Improved stability conditions for a class of stochastic Volterra-Levin equations," Applied Mathematics and Computation, vol. 231, pp. 39-47, 2014.

[8] T. A. Burton, "Fixed points and stability of a nonconvolution equation," Proceedings of the American Mathematical Society, vol. 132, no. 12, pp. 3679-3687, 2004.

[9] L. C. Becker and T. A. Burton, "Stability, fixed points and inverses of delays," Proceedings of the Royal Society of Edinburgh A: Mathematics, vol. 136, no. 2, pp. 245-275, 2006.

[10] C. H. Jin and J. W. Luo, "Stability of an integro-differential equation," Computers \& Mathematics with Applications, vol. 57, no. 7, pp. 1080-1088, 2009.

[11] A. Ardjouni, A. Djoudi, and I. Soualhia, "Stability for linear neutral integro-differential equations with variable delays," Electronic Journal of Differential Equations, vol. 2012, pp. 1-14, 2012.

[12] D. Zhao and S. Yuan, "3/2-stability conditions for a class of Volterra-Levin equations," Nonlinear Analysis: Theory, Methods \& Applications, vol. 94, pp. 1-11, 2014.

[13] J. Levin and A. Nohel, "Global asymptotic stability for nonlinear systems of differential equations and applications to reactor dynamics," Archive for Rational Mechanics and Analysis, vol. 5, pp. 194-211, 1960.

[14] T. A. Burton, "Fixed points, stability, and exact linearization," Nonlinear Analysis: Theory, Methods \& Applications, vol. 61, no. 5, pp. 857-870, 2005.

[15] D. Pi, "Study the stability of solutions of functional differential equations via fixed points," Nonlinear Analysis: Theory, Methods \& Applications, vol. 74, no. 2, pp. 639-651, 2011.

[16] D. Pi, "Stability conditions of second order integrodifferential equations with variable delay," Abstract and Applied Analysis, vol. 2014, Article ID 371639, 11 pages, 2014. 


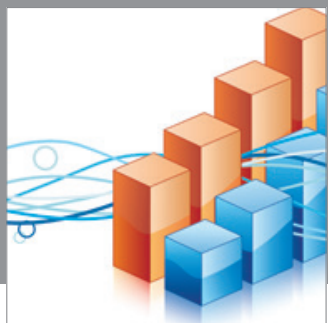

Advances in

Operations Research

mansans

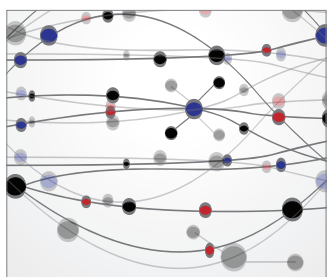

The Scientific World Journal
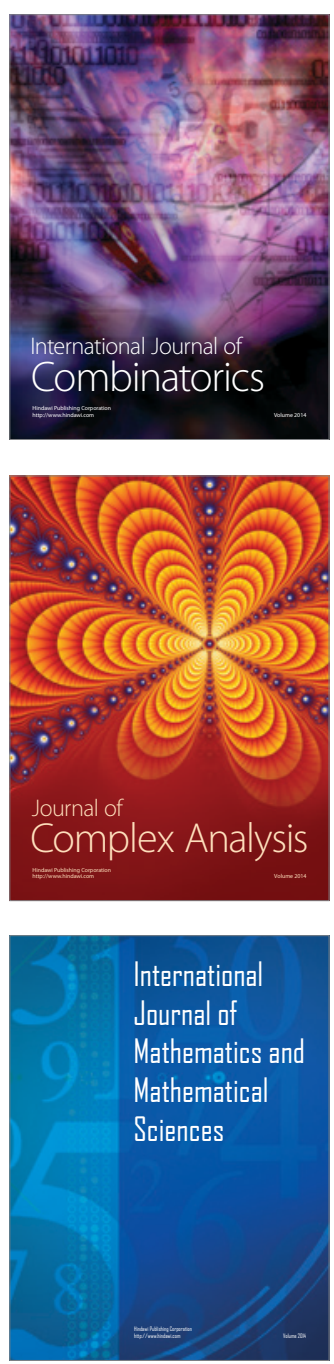
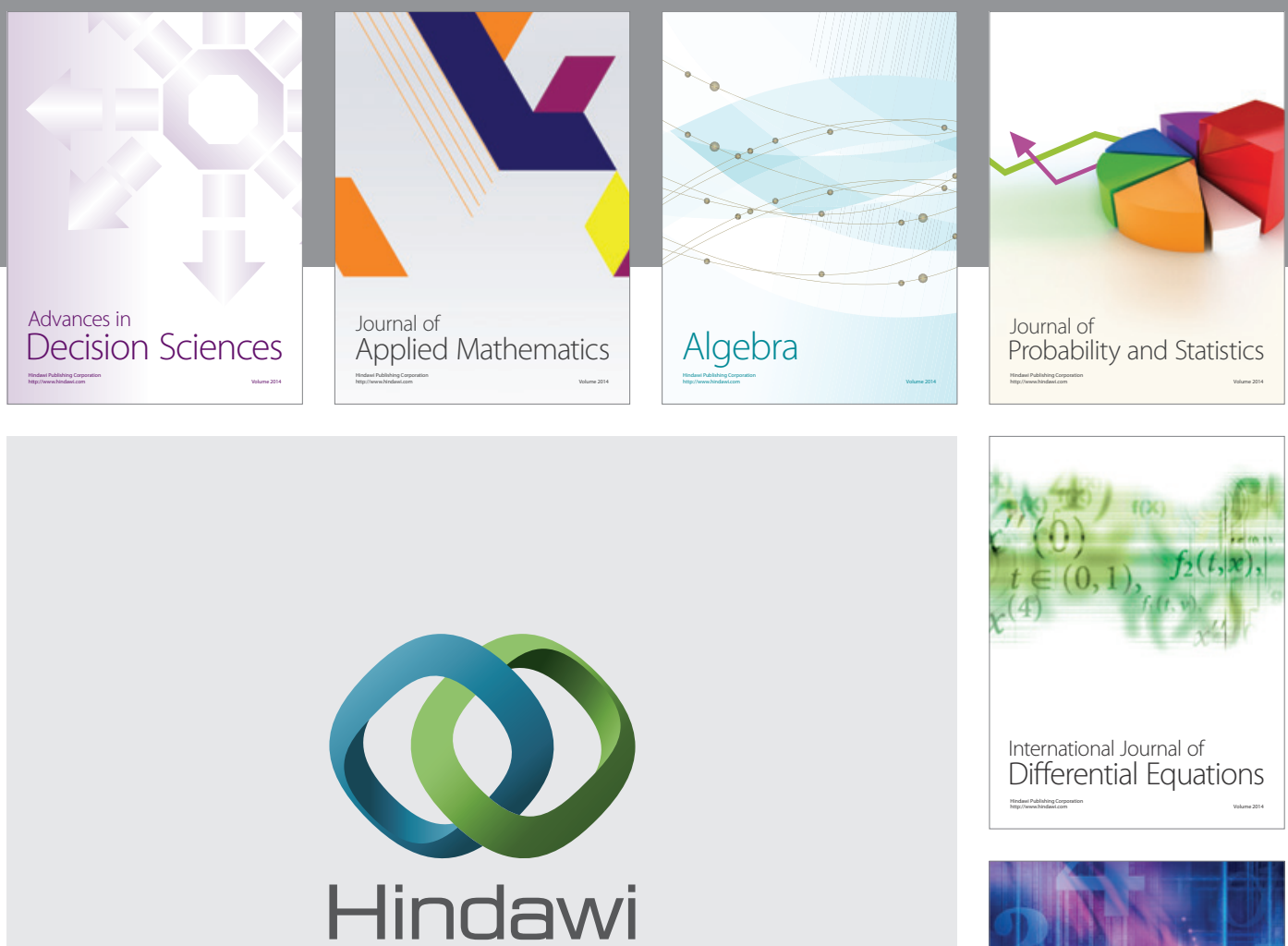

Submit your manuscripts at http://www.hindawi.com
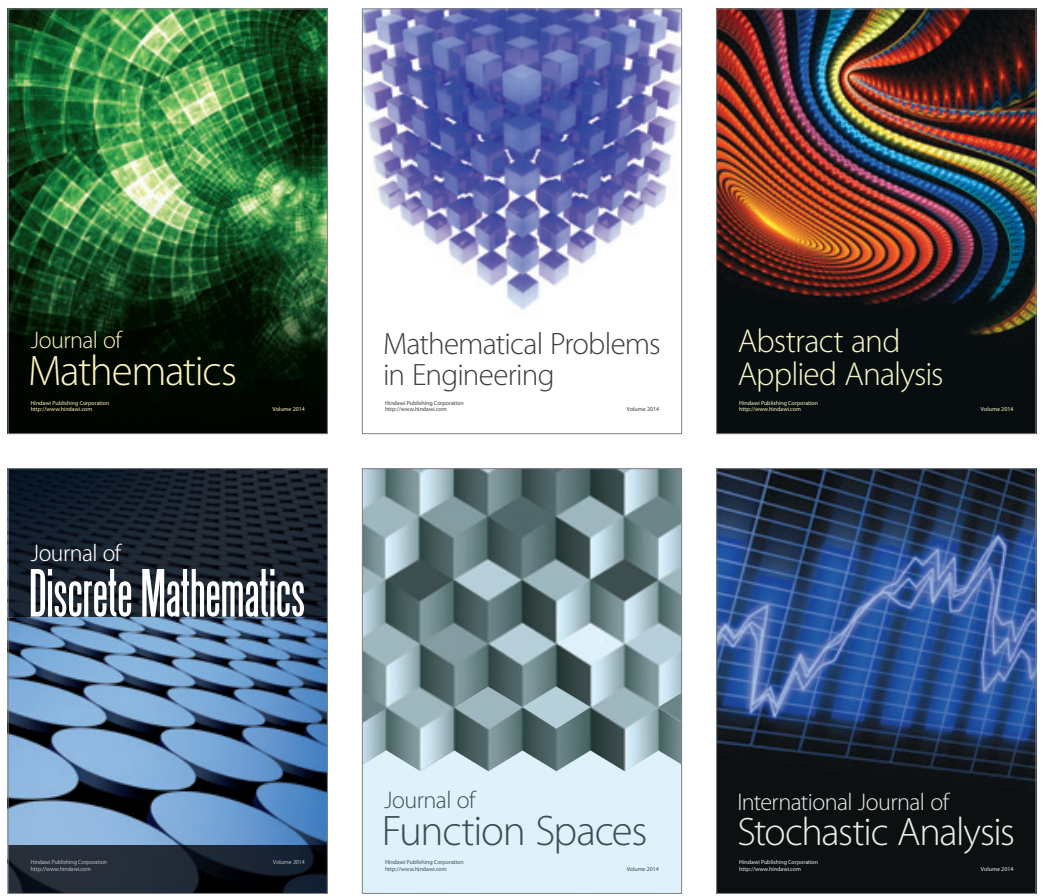

Journal of

Function Spaces

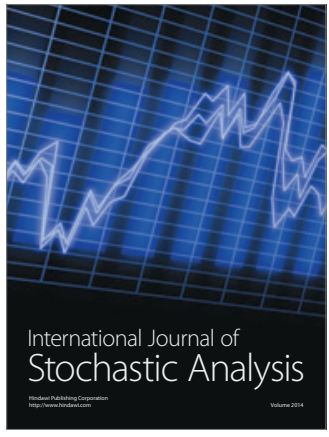

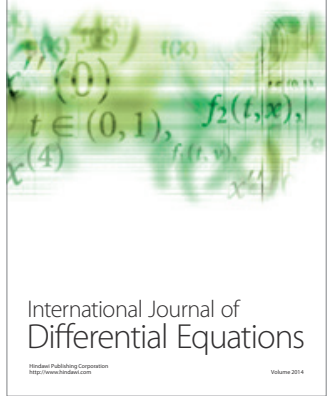
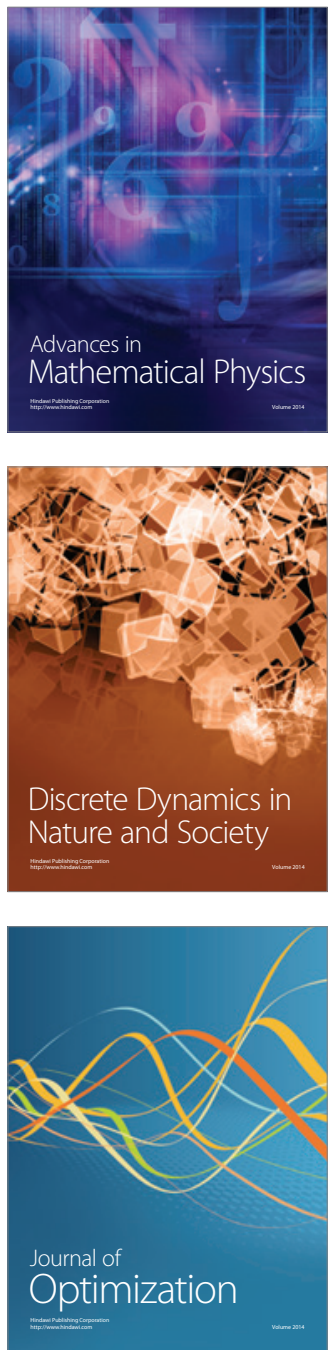